\title{
Spindle-configurations of skew lines
}

\author{
ROLAND BACHER \\ DAVID GARBER
}

\begin{abstract}
This paper is a contribution to the classification of configurations of skew lines, as studied mainly by Viro and his collaborators. We prove an improvement of a conjecture made by Crapo and Penne which characterizes isotopy classes of skew configurations with spindle-structure. By this result we can define an invariant, spindlegenus, for spindle-configurations.
\end{abstract}

57M25, 57Q37, 57Q45; 51H10, 57M27

\section{Introduction}

A configuration of $n$ skew lines in $\mathbb{R}^{3}$ or a skew configuration or an interlacing of $n$ skew lines is a set of $n$ non-intersecting lines in $\mathbb{R}^{3}$ containing no pair of parallel lines.

Two skew configurations $C_{1}$ and $C_{2}$ are isotopic if there exists a rigid isotopy (continuous deformation of skew configurations or, equivalently, isotopy of the ambient space under which all $n$ lines remain pairwise skew lines) from $C_{1}$ to $C_{2}$.

The study and classification of configurations of skew lines (up to isotopy) was initiated by Viro [16] and pursued by Viro, Mazurovskiĭ, Borobia-Mazurovskiı̌, Drobotukhina and Khashin, see for example Borobia-Mazurovskiı̌ [1; 2], Drobotukhina [4], Khashin [6], Mazurovskiǐ [11; 12] and Viro-Drobotukhina [17]. The original definitions, first invariants and classifications up to five lines are due to Viro. Mazurovskiı̆ classified configurations of six lines, see [11] and [12]. Borobia and Mazurovskiı̌ succeeded in classifying configurations of seven lines (see [1] and [2]) making use of a powerful invariant defined by Drobotukhina, see [4], who defined a Kauffman polynomial for such configurations. The numbers of distinct skew configurations, up to isotopy, having at most 7 lines are shown below.

\begin{tabular}{|c|c||c|c|}
\hline Lines & Isotopy classes & Lines & Isotopy classes \\
\hline 2 & 1 & 5 & 7 \\
3 & 2 & 6 & 19 \\
4 & 3 & 7 & 74 \\
\hline
\end{tabular}


The survey paper [17] (and its refreshed versions available on the author's website and on the arXiv) contains accurate historical information and is a good introduction to the subject and its higher-dimensional generalizations. Most of these results, with changes in terminology, are also exposed in the survey paper by Crapo and Penne [3]. Our choice of terminology is often influenced by it and differs thus sometimes from the terminology used by the authors of the original works. Finally let us cite Matei-Suciu [10] which is related to spindle configurations.

A spindle (or isotopy join or horizontal configuration) is a particularly nice configuration of skew lines in which all lines intersect an oriented additional line $A$, called the axis of the spindle. Its isotopy class is completely described by a spindle-permutation $\sigma:\{1, \ldots, n\} \longrightarrow\{1, \ldots, n\}$ encoding the order in which an open half-plane bounded by $A$ and revolving around it intersects the lines during a half-turn (see Section 5 for the precise definition). A spindle-configuration is a skew configuration isotopic to a spindle.

Consider the spindle-equivalence relation on permutations of $\{1, \ldots, n\}$ generated by transformations of the following three types.

(1) $\sigma \sim \mu$ if $\mu=\rho^{s} \sigma \rho^{t}$ for some integers $0 \leq s, t<n$ where $\rho$ is the cycle $(1,2, \ldots, n)$.

(2) $\sigma \sim \mu$ if there exists $k \leq n$ such that $\sigma(i) \leq k$ for $i \leq k$ and $\mu=\tau_{k} \sigma \tau_{k}$ where $\tau_{k}=(1, k)(2, k-1) \cdots$ is the involution fixing $i>k$ and transposing the elements $\{i, k+1-i\}$ for $i \leq k$.

(3) $\sigma \sim \mu$ if there exists $k \leq n$ such that $\sigma(i) \leq k$ for $i \leq k$ and

$$
\mu(i)= \begin{cases}\sigma^{-1}(i) & i \leq k \\ \sigma(i) & i>k .\end{cases}
$$

Our main result is an improvement of Crapo-Penne [3, Conjecture 59].

Theorem 1.1 Two spindle-permutations $\sigma, \sigma^{\prime}$ give rise to isotopic spindle-configurations if and only if $\sigma$ and $\sigma^{\prime}$ are spindle-equivalent.

Remark 1.2 The fact that spindle-equivalent permutations lead to isotopic spindles is [3, Theorem 62] for transformations of type (1) and (2) and follows from KhashinMazurovskiǔ [7, Theorem 3.2] for all three transformations. The original conjecture [3, Conjecture 59] is slightly weaker than the statement of Theorem 1.1. The formulation of Theorem 1.1 is however equivalent to [3, Conjecture 59] by [7, Theorem 3.2]. We will reprove the easy direction of Theorem 1.1 by simplifying (for transformations of 
type (3)) the known proofs which realize all transformations for spindle-equivalence by rigid isotopies. The proof of the other direction is the main result of this paper. Let us also mention that the proof does not exclude the existence of "exotic" isotopies: isotopies between spindles which are not reducible (up to deformations) to a finite sequence of rigid isotopies associated to transformation defining spindle-equivalence, see Remark 6.5.

Orienting and labeling all lines of a skew configuration, one gets a linking matrix by considering the signs of crossing lines. The associated switching class or homology equivalence class is independent of labels and orientations. A result of Khashin and Mazurovskiu [7, Theorem 3.2] states that homology-equivalent spindles (spindles defining the same switching class) are isotopic. We have thus the following Corollary.

Corollary 1.3 Two spindle permutations define the same switching class if and only if they are spindle-equivalent.

Isotopy classes of spindle-configurations have thus an easy combinatorial description and can be considered as "understood", either in terms of spindle-equivalence classes or in terms of switching classes, in contrast to the general case where no (provenly) complete invariants are available.

A few useful and mostly well-known invariants for configurations of skew lines follow.

(1) Equivalence classes of skew pseudoline diagrams (see Section 2): Completeness unknown (this is a major problem since the obvious planar representation of skew configurations is perhaps not faithful). A powerful combinatorial invariant somewhat tedious to handle. Switching classes and Kauffman polynomials factorize through it.

(2) Switching classes or two-graphs, see Zaslavsky [18, page 7], also called homological equivalence classes, see Borobia-Mazurovskir [2], are equivalent to the description of the sets of linking numbers or linking coefficients, see [2] or [17]. The definition of this invariant uses a linking matrix encoding the signs of oriented crossing lines (not intersecting in the compactification $\mathbb{R P} \mathbb{P}^{3} \supset \mathbb{R}^{3}$ ). The switching class is a complete invariant for configurations of up to 5 skew lines and is not complete for more than 5 lines. Switching-classes are however a complete invariant for spindle-configurations by [7, Theorem 3.2].

Slightly weaker (but more elementary to handle) than the switching class is the characteristic polynomial of a linking matrix $X$

$$
P_{X}(t)=\sum_{i=0}^{n} \alpha_{i} t^{i}=\operatorname{det}(t \mathrm{I}-X) \text {. }
$$


The coefficient $\alpha_{n-3}$ of $P_{X}(t)$ conveys the same information as the sum of linking numbers introduced by Viro.

(3) Kauffman polynomials: A powerful invariant introduced by Drobothukina in [4] which is unfortunately difficult to compute. Its completeness is unknown. See also [3, Section 14 and Appendix] for a list of computed examples.

(4) Link invariants for links in the 3-sphere $\mathbb{S}^{3}$ applied to the preimage $\pi^{-1}(C) \subset$ $\mathbb{S}^{3}$ of a skew configuration $C \subset \mathbb{R}^{3} \subset \mathbb{R} \mathbb{P}^{3}$ under the double covering $\pi: \mathbb{S}^{3} \longrightarrow$ $\mathbb{R} \mathbb{P}^{3}$. (This is called a Temari model by some authors, see for instance [3, Section 11].)

(5) Existence of a spindle structure. A generally very weak invariant since spindle structures are rare among configurations with many lines. Theorem 1.1 shows however that the spindle-equivalence class provides a complete invariant for the very small subset of skew configurations with a spindle structure. Moreover, the existence of a spindle structure in a given switching class can be decided algorithmically (work in progress by the authors).

(6) One should also mention the shellability order, a generalization of the notion of spindle structure, used as a classification tool in [2].

The sequel to the paper is organized as follows: Section 2 introduces skew pseudoline diagrams. Section 3 and Section 4 are devoted to switching classes.

Section 5 describes spindle-configurations and contains a proof of the easy (and known) direction in Theorem 1.1: Spindle-equivalent permutations yield isotopic configurations. Section 6 proves the difficult direction: Permutations associated to isotopic spindles are spindle-equivalent. This completes the proof of Theorem 1.1. Section 7 contains an easy proof of [7, Theorem 3.2]: Spindle configurations with switching-equivalent linking matrices are isotopic. This implies Corollary 1.3.

Section 8 describes a somewhat curious invariant for spindle-equivalence classes of permutations (or spindle-configurations) which involves 2-dimensional topology.

To our knowledge, only Section 6 and Section 8 contain new results. The remaining sections recall known facts and sometimes simplify their proofs.

\section{Skew pseudoline diagrams}

Skew pseudoline diagrams are combinatorial objects providing a convenient tool for studying configurations of skew lines. 
Definition 2.1 A pseudoline in $\mathbb{R}^{2}$ is a smooth simple curve representing a non-trivial cycle in $\mathbb{R P}^{2}$. An arrangement of $n$ pseudolines in $\mathbb{R}^{2}$ is a set of $n$ pseudolines with pairs of pseudolines intersecting transversally exactly once. An arrangement is generic if no triple intersections occur.

Definition 2.2 A skew pseudoline diagram of $n$ pseudolines in $\mathbb{R}^{2}$ is a generic arrangement of $n$ pseudolines with crossing data at intersections. The crossing data selects at each intersection the over-crossing pseudoline.

We draw skew pseudoline diagrams with the conventions used for knots and links: under-crossing curves are slightly interrupted at crossings.

Skew pseudoline diagrams are equivalent if they are related by a finite sequence of the following local moves, presented in Figure 1 (see [3, Section 9]).
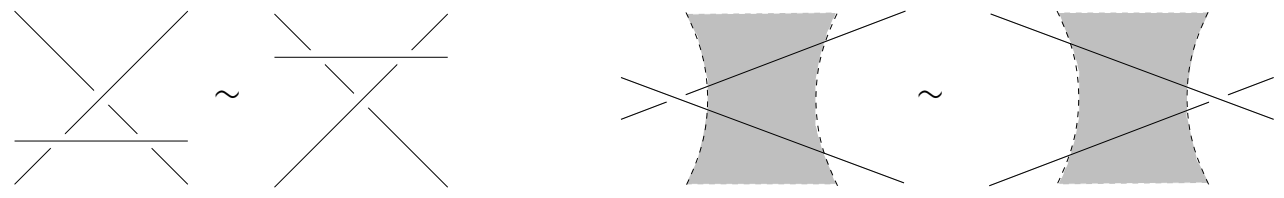

Figure 1: Local moves

(1) Reidemeister-3 (or $*$-move), presented on the left half of Figure 1, the most interesting of the three classical moves for knots and links.

(2) Projective move (or $\|$-move): pushing a crossing through infinity as presented on the right half of Figure 1 (with the grey region enclosed by the dashed hyperbola containing all remaining lines).

Generic projections of isotopic skew configurations yield equivalent skew pseudoline diagrams (see for instance [3, Theorem 48]).

Not every skew pseudoline diagram is equivalent to the projection of a suitable skew configuration: a configuration involving at least 4 skew lines is never equivalent to an alternating skew pseudoline diagram (see Pach-Pollack-Welzl [13]). There are even examples of generic diagrams having $n \geq 9$ pseudolines which cannot be realized using straight lines, see Grünbaum [5].

The existence of non-isotopic skew configurations inducing equivalent skew pseudoline diagrams cannot be excluded. This is a major difficulty for classification, see [3, Section 17, Problem 2]. 


\section{Linking matrices and switching classes}

One assigns signs to pairs of oriented under- or over-crossing curves (as arising for instance from oriented knots and links) which are drawn in the oriented plane. Figure 2 shows a positive and a negative crossing.
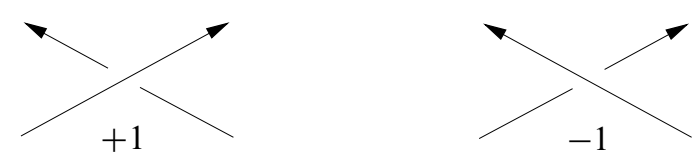

Figure 2: Positive and negative crossings, denoted by \pm 1

The sign or linking number $\operatorname{lk}\left(L_{A}, L_{B}\right)$ between two oriented skew lines $L_{A}, L_{B} \subset \mathbb{R}^{3}$ was introduced by Viro in [16] and can be computed as follows: choose ordered pairs of points $\left(A_{\alpha}, A_{\omega}\right)$ on $L_{A}$ (resp. $\left(B_{\alpha}, B_{\omega}\right)$ on $\left.L_{B}\right)$ which induce the orientations. Then the sign of the crossing determined by $L_{A}$ and $L_{B}$ is given by

$$
\operatorname{lk}\left(L_{A}, L_{B}\right)=\operatorname{sign}\left(\operatorname{det}\left(\begin{array}{c}
A_{\omega}-A_{\alpha} \\
B_{\alpha}-A_{\omega} \\
B_{\omega}-B_{\alpha}
\end{array}\right)\right) \in\{ \pm 1\}
$$

where $\operatorname{sign}(x)=\frac{x}{|x|}$ for $x \neq 0$.

Signs of crossings are also defined in skew pseudoline diagrams.

The linking matrix of a diagram of $n$ oriented and labeled skew pseudolines $L_{1}, \ldots, L_{n}$ is the symmetric $n \times n$ matrix $X$ with diagonal coefficients $x_{i, i}=0$ and $x_{i, j}=$ $\operatorname{lk}\left(L_{i}, L_{j}\right)$ for $i \neq j$.

Example 3.1 Figure 3 shows a labeled and oriented configuration of six skew lines with linking matrix

$$
X=\left(\begin{array}{rrrrrr}
0 & -1 & -1 & 1 & 1 & -1 \\
-1 & 0 & 1 & 1 & 1 & 1 \\
-1 & 1 & 0 & 1 & -1 & -1 \\
1 & 1 & 1 & 0 & 1 & -1 \\
1 & 1 & -1 & 1 & 0 & 1 \\
-1 & 1 & -1 & -1 & 1 & 0
\end{array}\right)
$$

having characteristic polynomial $\operatorname{det}(t \mathrm{I}-X)=\left(t^{2}-5\right)^{3}$.

Two symmetric matrices $X$ and $Y$ are switching-equivalent if

$$
Y=D P^{t} X P D
$$




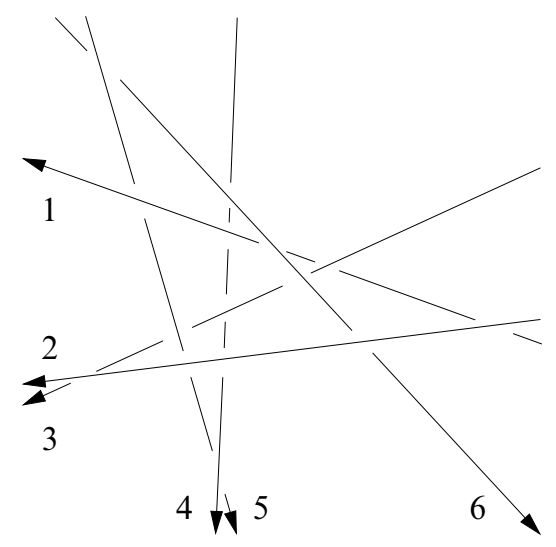

Figure 3: A configuration of 6 labeled and oriented skew lines

where $P$ is a permutation matrix and $D$ is a diagonal matrix with diagonal entries in $\{ \pm 1\}$. Since $P D$ is an orthogonal matrix, we have $(P D)^{-1}=D P^{t}$ and switchingequivalent matrices are thus conjugate.

Proposition 3.2 All linking matrices of a fixed skew pseudoline diagram are switchingequivalent.

Proof Relabeling the lines conjugates a linking matrix $X$ by a permutation matrix. Reversing the orientation of some lines amounts to conjugation by a diagonal matrix with diagonal entries in $\{ \pm 1\}$.

Remark 3.3 The terminology switching classes (also called two-graphs) is motivated by the switching-relation for simple graphs, see for instance Zaslavsky [18].

Remark 3.4 Characteristic polynomials separate switching classes only for orders $n \leq 7$. For higher orders there exist cospectral pairs of switching classes.

Remark 3.5 Mazurovskiu proved that the switching class is a complete invariant for isotopy classes of skew configurations having fewer than 6 lines.

Moreover, Mazurovskiĭ discovered that the skew configuration of 6 lines depicted in Figure 3 and its mirror (obtained by changing the signs of all crossings) are in the same switching class (represented by the matrix $X$ or $-X$ of Example 3.1) but have different Kauffman polynomials and are thus not isotopic, see [12, Theorem 3.2.1]. 
All $2^{\left(\begin{array}{c}n \\ 2\end{array}\right)}$ possible linking matrices can be obtained from a fixed skew pseudoline diagram by an appropriate choice of crossing data. The number of equivalence classes of skew pseudoline diagrams with $n$ pseudolines equals thus at least the number of switching classes of order $n$. Remark 3.5 shows that this inequality is in general strict. Moreover, the number $N_{S C}(n)$ of switching classes of order $n$ satisfies the inequality

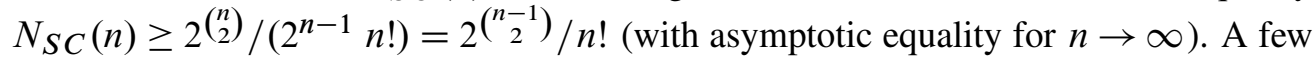
values for these numbers $N_{S C}(n)$, together with the numbers of distinct characteristic polynomials associated to switching classes are gathered in the following table.

\begin{tabular}{|c|c|c|}
\hline Lines & $\begin{array}{c}\text { Characteristic } \\
\text { polynomials }\end{array}$ & $\begin{array}{c}\text { Switching } \\
\text { classes }\end{array}$ \\
\hline 6 & 16 & 16 \\
7 & 54 & 54 \\
8 & 235 & 243 \\
9 & 1824 & 2038 \\
\hline
\end{tabular}

One can use representation theory of the symmetric groups in order to derive a formula for $N_{S C}(n)$ (see Mallows-Sloane [8] and Sequence A2854 in Sloane [15]).

The map

$$
\text { \{configurations of skew lines\} } \longrightarrow \text { \{switching classes\} }
$$

is perhaps not surjective in general (there seems to be an unpublished counterexample by Peter Shor for $n=71$, see [3, Section 3]). We checked however that all 243 switching classes of order 8 arise as linking matrices of skew configurations, as claimed in [3, Theorem 5]. (The corresponding result holds also for fewer lines.) There are thus at least 243 isotopy classes of configurations containing 8 skew lines.

The mirror configuration $\bar{C}$ obtained by reflecting a skew configuration $C$ through the $z=0$ plane has opposite crossing data. We have thus $\bar{X}=-X$ for associated linking matrices $X$ and $\bar{X}$.

A configuration $C$ is amphicheiral if it is isotopic to its mirror $\bar{C}$. We cite without proof the following easy result, due to Viro, see [16] or [17].

\section{Proposition 3.6}

(i) The linking matrix $X$ of an amphicheiral configuration of skew lines is switchingequivalent to $-X$. In particular, amphicheiral configurations containing an odd number of skew lines have non-invertible linking matrices.

(ii) Amphicheiral configurations with $n \equiv 3(\bmod 4)$ lines do not exist. 


\section{Switching classes, vorticity and signature}

The set of vorticities (also called homological equivalence class or chiral signature), introduced by Viro in [16], is a classical and well-known invariant for skew pseudoline diagrams. We sketch below briefly the well-known proof that it corresponds to the switching class of an associated linking matrix.

In this paper we work with switching classes mainly because they are easier to handle. The vorticity $\operatorname{vort}\left(L_{i}, L_{j}, L_{k}\right)$ of three lines (see [16, Section 2] or [3, Section 3]) is defined as the product $x_{i, j} x_{j, k} x_{k, i} \in\{ \pm 1\}$ of the signs for the corresponding three crossings. The result is independent of the chosen orientations for $L_{i}, L_{j}, L_{k}$. It classifies the skew-configuration $\left\{L_{i}, L_{j}, L_{k}\right\}$ up to isotopy and yields an invariant

$\{$ triplets of lines in skew pseudoline diagrams $\} \longrightarrow\{ \pm 1\}$.

Let us remark that almost all authors use the terminology linking coefficient instead of vorticity. This is slightly confusing since the linking coefficient denotes also the isotopy type of a pair of oriented skew lines.

The set of vorticities is the list of vorticities $\operatorname{vort}\left(L_{i}, L_{j}, L_{k}\right)$ for all triplets of lines $\left\{L_{i}, L_{j}, L_{k}\right\}$ in a skew pseudoline diagram.

Sets of vorticities (defining a two-graph, see [18]) and switching classes are equivalent. Indeed, vorticities of a skew pseudo-line diagram $D$ can easily be retrieved from a linking matrix for $D$. Conversely, given all vorticites $\operatorname{vort}\left(L_{i}, L_{j}, L_{k}\right)$ of a diagram $D$, choose an orientation of the first line $L_{1}$. Orient the remaining lines $L_{2}, \ldots, L_{n}$ such that they cross $L_{1}$ positively. A linking matrix $X$ for $D$ is given by $x_{1, i}=x_{i, 1}=$ $1,2 \leq i \leq n$ and $x_{a, b}=\operatorname{vort}\left(L_{1}, L_{a}, L_{b}\right)$ for $2 \leq a \neq b \leq n$.

Two skew pseudoline diagrams are homologically equivalent (the terminology refers to properties of the complement, endowed with a suitable extra-structure, of a configuration in $\left.\mathbb{R P}^{3}\right)$ if there exists a bijection between their lines, which preserves all vorticities. Two diagrams are homologically equivalent if and only if they have switching-equivalent linking matrices.

Some authors (see [3, Section 3 and Appendix]) consider the chirality $\left(\gamma_{+}, \gamma_{-}\right)$defined as

$$
\begin{aligned}
& \gamma_{+}=\sharp\left\{1 \leq i<j<k \leq n \mid \operatorname{vort}\left(L_{i}, L_{j}, L_{k}\right)=1\right\} \\
& \gamma_{-}=\sharp\left\{1 \leq i<j<k \leq n \mid \operatorname{vort}\left(L_{i}, L_{j}, L_{k}\right)=-1\right\}
\end{aligned}
$$

of a skew pseudoline diagram. One has $2 \gamma_{+}=\left(\begin{array}{l}n \\ 3\end{array}\right)+c, 2 \gamma_{-}=\left(\begin{array}{l}n \\ 3\end{array}\right)-c$ where

$$
c=\sum_{1 \leq i<j<k \leq n} x_{i, j} x_{j, k} x_{k, i}=\frac{1}{6} \operatorname{trace}\left(X^{3}\right)=-\frac{\alpha_{n-3}}{2}
$$


is proportional to the coefficient of $t^{n-3}$ in the characteristic polynomial $\operatorname{det}(t I-X)=$ $\sum_{i=0}^{n} \alpha_{i} t^{i}$ of a linking matrix $X$.

Remark 4.1 There is a canonical choice (obtained by reversing all rows and columns containing initially an odd number of 1 's and $(-1)$ 's and closely related to simple Eulerian graphs, see Seidel [14]) avoiding the sign indeterminancy for linking matrices representing switching classes of odd order. For even orders, there seems to be no completely satisfactory analogue.

\section{Spindles}

\subsection{Spindle-configuration}

Mazurovskil introduced a construction, called the isotopy join, which associates a configuration of $\left(a_{1}+a_{2}+1\right)$-dimensional affine subspaces in $\mathbb{R}^{n_{1}+n_{2}+1}$ to pairs of configurations of $a_{i}$-dimensional affine subspaces in $\mathbb{R}^{n_{i}}$, see for instance [7, Section 1.7]. The special case $a_{1}=a_{2}=0$ and $n_{1}=n_{2}=1$ yields configurations of skew lines, called spindles by some authors. We recall that a spindle is a configuration of skew lines intersecting an oriented auxiliary line $A$, called its axis. A spindle configuration (or a spindle structure) is a configuration of skew lines isotopic to a spindle.

The orientation of the axis $A$ induces a linear order $L_{1}<\cdots<L_{n}$ on the $n$ lines of a spindle $C$. Each line $L_{i} \in C$ defines a plane $\Pi_{i}$ containing $L_{i}$ and the axis $A$.

A second auxiliary line $B$ (called a directrix) in general position with respect to $A, \Pi_{1}, \ldots, \Pi_{n}$ and oriented in order to cross $A$ negatively, intersects the planes $\Pi_{1}, \ldots, \Pi_{n}$ at points $\sigma\left(L_{i}\right)=B \cap \Pi_{i}$. One can assume $\sigma\left(L_{i}\right) \in L_{i}$ by a suitable rotation fixing $A \cap \Pi_{i}$ of the plane $\Pi_{i}$ containing $L_{i}$. Since the orientation of $B$ induces a linear order on the points $\sigma\left(L_{i}\right)$, we get a spindle-permutation (still denoted) $i \longmapsto \sigma(i)$ of the set $\{1, \ldots, n\}$ by identifying the two linearly ordered sets $L_{1}, \ldots, L_{n}$ and $\sigma\left(L_{1}\right), \ldots, \sigma\left(L_{n}\right)$ in the obvious way with $\{1, \ldots, n\}$. Figure 4 displays an example corresponding to $\sigma(1)=1, \sigma(2)=4, \sigma(3)=2, \sigma(4)=5, \sigma(5)=3$. In the sequel, we often denote by $(\alpha, \beta)$ the line $L_{\alpha}$ defined by $\sigma(\alpha)=\beta$ in a spindle configuration. The spindle of Figure 4 thus consists of the lines $(1,1),(2,4),(3,2),(4,5)$ and $(5,3)$.

Spindles can also be represented by (the isotopy classes of) configurations of skew lines with all lines contained in distinct affine horizontal planes of $\mathbb{R}^{3}$ (horizontal configurations). An associated spindle permutation encodes the two orders on the set of lines given by the heights of the horizontal planes containing them and by the slopes of the lines after orthogonal projection onto such a horizontal plane. 


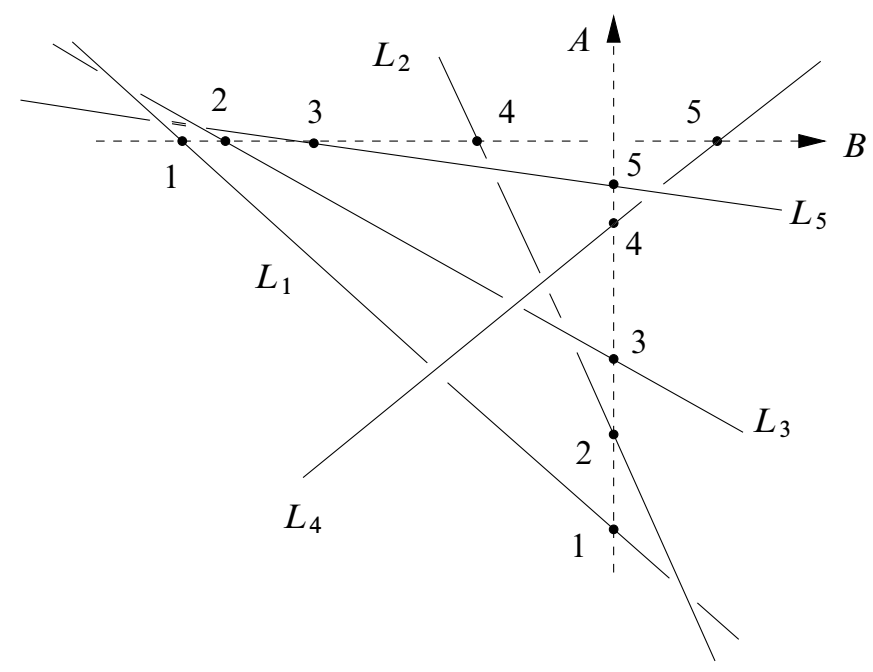

Figure 4: A spindle

A linking matrix $X$ of a spindle $C$ is easily computed as follows. Transform $C$ isotopically into a spindle with oriented axis $A$ and directrix $B$ as above. Orient a line $L_{i}$ from $L_{i} \cap A$ to $L_{i} \cap B$. A straightforward computation shows that the linking matrix $X$ of this labeled and oriented skew configuration has coefficients

$$
x_{i, j}=\operatorname{sign}((i-j)(\sigma(i)-\sigma(j)))
$$

where $\operatorname{sign}(0)=0$ and $\operatorname{sign}(x)=\frac{x}{|x|}$ for $x \neq 0$ and where $\sigma$ is the corresponding spindle-permutation. For example, the configuration of Figure 4 has linking matrix

$$
X=\left(\begin{array}{rrrrr}
0 & 1 & 1 & 1 & 1 \\
1 & 0 & -1 & 1 & -1 \\
1 & -1 & 0 & 1 & 1 \\
1 & 1 & 1 & 0 & -1 \\
1 & -1 & 1 & -1 & 0
\end{array}\right) .
$$

Remark 5.1 A configuration $C$ of $n$ skew lines has a spindle structure if and only if its mirror configuration $\bar{C}$ has a spindle structure. A spindle permutation $\bar{\sigma}$ for $\bar{C}$ is then for instance given by $\bar{\sigma}(i)=n+1-\sigma(i), 1 \leq i \leq n$, where $\sigma$ is a spindlepermutation for $C$. For $n \leq 13$ such that $n \not \equiv 3(\bmod 4)$, the table of Section 5.2 shows the existence of spindle configurations having $n$ lines which are amphicheiral (isotopic to their mirror). 


\subsection{Spindle-equivalent permutations}

The aim of this subsection is to show that the three types of transformations of spindlepermutations, defined by Crapo and Penne in [3, Section 15], preserve the associated spindle configuration, up to isotopy. This is the easy direction in Theorem 1.1. This part is also proved in [7, Theorem 3.2].

A (linear) block of size $k$ or a $k$-block in a permutation $\sigma$ of $\{1, \ldots, n\}$ is a subset $\{i+1, \ldots, i+k\}$ of $k$ consecutive integers in $\{1, \ldots, n\}$ such that

$$
\sigma(\{i+1, \ldots, i+k\})=\{j+1, \ldots, j+k\}
$$

for some integer $j$ (that is the image under $\sigma$ of a set $\{i+1, \ldots, i+k\}$ of $k$ consecutive integers is again a set of $k$ consecutive integers). In the sequel, we denote by $[\alpha, \beta]=\{\alpha, \alpha+1, \ldots, \beta-1, \beta\} \subset\{1, \ldots, n\}$ a subset of consecutive integers and by $\sigma([i+1, i+k])=[j+1, j+k]$ a $k$-block as above.

Recall that two spindle-permutations are equivalent (see [3, Section 15]) if they are equivalent under the equivalence relation generated by the following spindle moves:

(1) (Circular move)

$$
\sigma \sim \mu \text { if } \mu=\rho^{s} \sigma \rho^{t}
$$

for some integer $0 \leq s, t<n$ where $\rho$ is the $n$-cycle $(1,2, \ldots, n)$.

(2) (Vertical reflection of a block or local reversal) $\sigma \sim \mu$ if there exists an integer $1<k \leq n$ such that $\sigma([1, k])=[1, k]$ and

$$
\mu(i)=\left\{\begin{array}{ll}
k+1-\sigma(k+1-i) & i \leq k \\
\sigma(i) & i>k
\end{array} .\right.
$$

(see Figure 5).
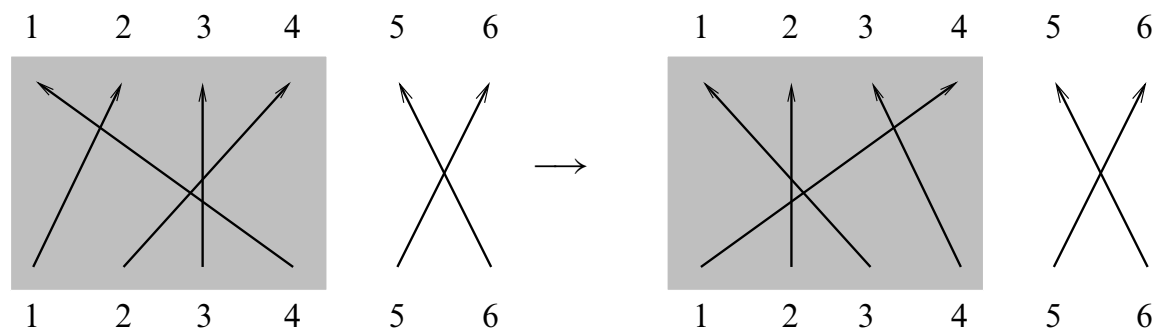

Figure 5: Vertical reflection of a block 
(3) (Horizontal reflection of a block or local inversion) $\sigma \sim \mu$ if there exists an integer $1<k \leq n$ such that $\sigma([1, k])=[1, k]$ and

$$
\mu(i)= \begin{cases}\sigma^{-1}(i) & i \leq k \\ \sigma(i) & i>k\end{cases}
$$

(see Figure 6).
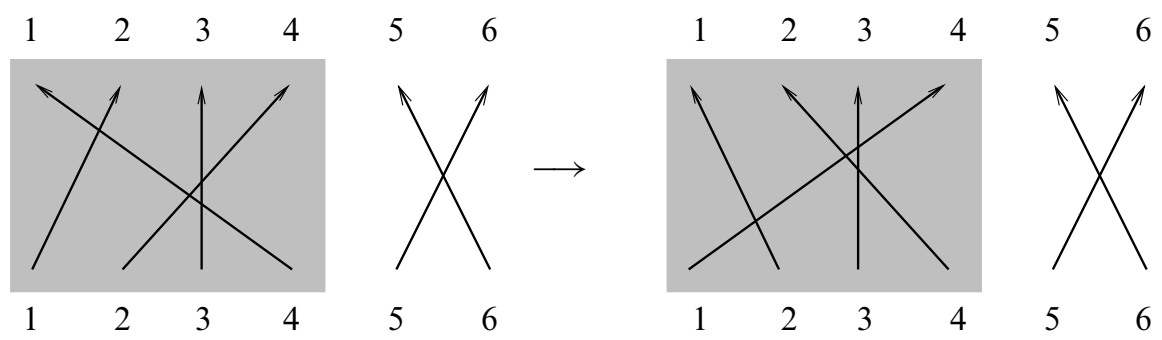

Figure 6: Horizontal reflection of a block

Circular moves suggest to extend the linear order on $\{1, \ldots, n\}$ to the cyclic order induced by the compactification $\mathbb{R P}^{2} \supset \mathbb{R}$. We have an obvious notion of cyclic blocks and can consider horizontal and vertical reflections of cyclic blocks related to vertical and horizontal reflections as above by conjugations involving circular moves. Such (more general) moves lead to the same equivalence relation as the three moves considered above and we allow them for the sake of conciseness.

The following table shows the number of spindle-permutations, up to equivalence, for $n \leq 13$ (see Sequence A110888 in [15]). We also indicate the number of amphicheiral spindle-permutations (giving rise to amphicheiral skew configurations), up to equivalence (see Sequence A110890 in [15]).

\begin{tabular}{|c|c|c||c|c|c|}
\hline$n$ & spindle classes & amphicheiral classes & $n$ & spindle classes & amphicheiral classes \\
\hline 1 & 1 & 1 & 8 & 180 & 12 \\
2 & 1 & 1 & 9 & 985 & 5 \\
3 & 2 & 0 & 10 & 6867 & 83 \\
4 & 3 & 1 & 11 & 60108 & 0 \\
5 & 7 & 1 & 12 & 609112 & 808 \\
6 & 15 & 3 & 13 & 6909017 & 47 \\
7 & 48 & 0 & & & \\
\hline
\end{tabular}

Assertion (ii) of Proposition 3.6 explains the non-existence of amphicheiral classes for $n \equiv 3(\bmod 4)$. 
Remark 5.2 The number of spindle classes is a lower bound for the number of skewline configurations by Theorem 1.1, and for the number of distinct switching classes containing a linking matrix of a skew-line configuration by Corollary 1.3.

We restate and reprove the easy direction (see also [7]) of Theorem 1.1.

Proposition 5.3 Equivalent spindle-permutations yield isotopic spindle configurations.

Proof A transformation of type (1) amounts to pushing the last few lines of the spindle on the axis and directrix through infinity. This can be done isotopically.

An isotopy inducing a vertical reflection (type (2) above) can be described as follows: Consider the two complementary subblocks $\sigma([1, k])=[1, k]$ and $\sigma([k+1, n])=$ $[k+1, n]$ in $\sigma$. All lines of the first block $\sigma([1, k])=[1, k]$ can be squeezed isotopically into the interior of a small one-sheeted hyperboloid $H$ whose axis of revolution intersects orthogonally the axis $A$ and the directrix $B$ of $\sigma$. Moreover, we may assume that no line of the complementary block $\sigma([k+1, n])=[k+1, n]$ intersects the interior of $H$. The isotopy of skew lines given by rotating the interior (containing the block $\sigma([1, k])=[1, k])$ of $H$ by a half-turn around its axis of revolution induces a vertical reflection (see Figure 7).

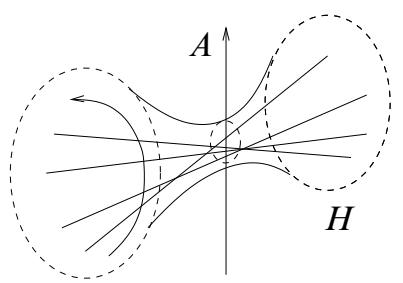

Figure 7: Isotopy for a transformation of type (2)

For constructing an isotopy inducing a horizontal reflection (type (3)) we start as above by pushing the $k$ lines of the first block $\sigma([1, k])=[1, k]$ into a small hyperboloid $H_{1}$ with revolution axis $C_{1}$ intersecting the axis $A$ and the directrix $B$ orthogonally. Denote by $I_{B}$ the open segment of $B$ contained in the interior of $H_{1}$. Moreover, suppose that the directions of the axis $A$ and the directrix $B$ are orthogonal. Push the lines of the complementary block $\sigma([k+1, n])=[k+1, n]$ in the positive sense along the directrix $B$ until they can be squeezed into the interior of a small revolution hyperboloid $H_{2}$ not intersecting $H_{1}$ with revolution axis $C_{2}$ parallel to the directrix $B$ of $\sigma$.

Rotate the interior of the first hyperboloid $H_{1}$ containing the block $\sigma([1, k])=[1, k]$ by a half-turn around the revolution axis $\mathrm{C}_{2}$ of $\mathrm{H}_{2}$ (see part (1) of Figure 8) and call 
the resulting hyperboloid $H_{1}^{\prime}$. Finally, rotate the hyperboloid $H_{1}^{\prime}$ and the lines inside it by $\pi / 2$ around its revolution axis $C_{1}^{\prime}$ and translate it along $C_{1}^{\prime}$ until the image of $I_{B}$ is contained in the directrix $A$ of $\sigma$ (see part (2) of Figure 8). This yields a spindle configuration whose spindle-permutation is related by a circular move and a horizontal reflection (and perhaps a vertical reflection of the first block, depending on the sense of the half-turn around $C_{1}^{\prime}$ ) to the initial spindle-permutation.

(1)

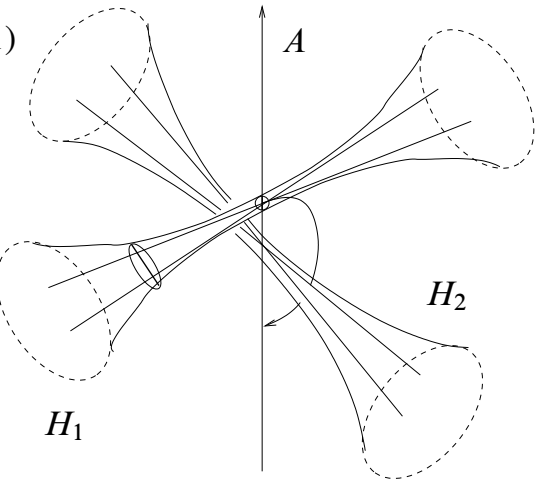

(2)

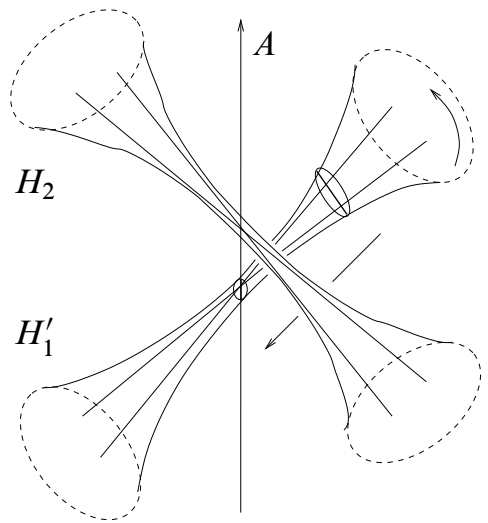

Figure 8: Isotopy for a transformation of type (3)

\section{Isotopic spindle configurations}

In this section, we prove the difficult direction of Theorem 1.1. We start by describing all blocks (up to spindle moves) in spindle configurations.

Proposition 6.1 Let $\sigma$ be a spindle-permutation with linking matrix $X$. Let $I$ be a subset of at least 2 indices corresponding to lines such that for all $i_{1}, i_{2} \in I$ and all $j \notin I$, the product

$$
x_{i_{1}, i_{2}} x_{i_{1}, j} x_{i_{2}, j} \in\{ \pm 1\}
$$

depends only on $i_{1}, i_{2}$ and is independent of $j$.

Then, up to spindle moves of $\sigma$, the lines $\left\{L_{i}\right\}_{i \in I}$ corresponding to I form a block of $\sigma$.

Corollary 6.2 Let $B \subset \sigma$ be a block of a spindle-permutation $\sigma$. Let $\iota$ be an isotopy between $\sigma$ and a second spindle-permutation $\sigma^{\prime}$. Then, up to spindle moves of $\sigma^{\prime}$, the set of lines $\iota(B) \subset \sigma^{\prime}$ is a block too. 
Proof of Proposition 6.1 We index the rows and columns of $X$ by $\{1, \ldots, n\}$ and first consider the case $\sharp(I)=2$. Up to circular moves, we can assume that $I=\{1, \alpha\}$ corresponds to the lines $(1,1),(\alpha, \beta) \in \sigma$. Up to replacing $\sigma$ by a mirror permutation $\bar{\sigma}$, we can also assume $x_{1, \alpha} x_{1, \gamma} x_{\alpha, \gamma}=1$ for $\gamma \in\{2, \ldots, n\} \backslash\{\alpha\}$. This implies $(\alpha-$ $\gamma)(\beta-\sigma(\gamma))>0$ and shows $\alpha=\beta$. Moreover, the lines $(2, \sigma(2)),(3, \sigma(3)), \ldots,(\alpha-$ $1, \sigma(\alpha-1)),(\alpha, \alpha)$ form a block $B \subset \sigma$ and a vertical reflection with respect to $B$ transforms the line $(\alpha, \alpha)$ into the line $(2,2)$.

Suppose now that the subset $I$ containing $k \geq 3$ lines is minimal in the sense that it contains no strict subset $I^{\prime} \subset I$ of $k^{\prime} \geq 2$ indices satisfying the condition of Proposition 6.1. Then we claim that the corresponding subset of lines $\{(i, \sigma(i))\}_{i \in I}$ form a block of $\sigma$. Indeed, otherwise there exist (up to a horizontal reflection and a circular move) indices $1<j_{1}<i<j_{2} \leq n$ with $1, i \in I$ and $j_{1}, j_{2} \notin I$. Up to replacing $\sigma$ by a mirror-permutation and up to circular moves we can assume $1=\sigma(1)<\sigma\left(j_{1}\right)<\sigma\left(j_{2}\right)$. The equality $x_{1, i} x_{1, j_{1}} x_{i, j_{1}}=x_{1, i} x_{1, j_{2}} x_{i, j_{2}}$ implies now $\sigma\left(j_{1}\right)<\sigma(i)<\sigma\left(j_{2}\right)$ for all $i \in I, j_{1}, j_{2} \notin I$ with $j_{1}<i<j_{2}$. A similar argument shows that $\sigma(i) \notin\left\{\sigma\left(j_{1}\right), \sigma\left(j_{1}\right)+1, \ldots, \sigma\left(j_{2}\right)\right\}$ for all $i \in I$ with $i<j_{1}$ or $i>j_{2}$. This implies that both sets $I_{1}=I \cap\left(\left\{1,2, \ldots, j_{1}-1\right\} \cup\left\{j_{2}+1, \ldots, n\right\}\right)$ and $I_{2}=I \cap\left\{j_{1}+1, \ldots, j_{2}-1\right\}$ satisfy the condition of Proposition 6.1. At least one of the non-empty proper subsets $I_{1}, I_{2} \subset I$ contains 2 elements or more and this contradicts the assumed minimality of $I$.

In the general case, we consider a proper subset $I^{\prime} \subset I$ which satisfies the condition of Proposition 6.1 and which contains either only two lines or is minimal as defined previously. Since Proposition 6.1 holds for $I^{\prime}$ we can suppose (up to spindle moves if $\sharp\left(I^{\prime}\right)=2$ ) that the lines of $I^{\prime}$ form a block $B^{\prime} \subset \sigma$. Considering the lines of $B^{\prime}$ as rigidly linked (and thus allowing only spindle-moves of $\sigma$ transforming all lines of $B^{\prime}$ similarly) we can consider $B^{\prime}$ as being represented by a single line $L^{\prime} \in B^{\prime}$. This yields a smaller spindle-permutation $\tilde{\sigma}$ together with a subset of indices $\tilde{I}$ satisfying the condition of Proposition 6.1 for the corresponding linking matrix $\tilde{X}$ obtained by removing from $X$ all rows and columns corresponding to $B^{\prime} \backslash L^{\prime}$. Proposition 6.1 holds now for $\widetilde{\sigma}$ by induction on the number of lines and gluing back the rigid block $B^{\prime}$ (its insertion is well-defined up to a vertical and horizontal reflection of $B^{\prime}$ ) onto $L^{\prime} \in \widetilde{\sigma}$ yields the result.

Proof of Corollary 6.2 The set of indices corresponding to a subblock $B \subset \sigma$ satisfies the condition of Proposition 6.1 for a common linking matrix $X$ of the isotopic spindles $\sigma$ and $\sigma^{\prime}$. 
Remark 6.3 The proof of Proposition 6.1 shows that blocks of more than 2 lines which are minimal (contain no non-trivial proper subblock) cannot be "torn apart" by spindle moves.

Theorem 6.4 Let $\iota$ be an isotopy relating two spindle configurations (associated to spindle-permutations) $\sigma$ and $\sigma^{\prime}$. Then the bijection from the lines of $\sigma$ onto the lines of $\sigma^{\prime}$ induced by $\iota$ can be realized by spindle moves.

Proof of Theorem 1.1 Follows from Proposition 5.3 and Theorem 6.4.

Remark 6.5 Theorem 6.4 does not exclude the existence of "exotic" isotopies between spindle-equivalent spindle configurations which do not arise from (a continuous deformation of a sequence of) spindle moves.

For proving Theorem 6.4 we have to generalize some notions for spindle-permutations: A spindle-permutation is a bijection $\sigma: E \longrightarrow F$ between two finite subsets $E, F \subset \mathbb{R}$ which we consider either linearly ordered or cyclically ordered by the cyclic order induced by $\mathbb{R}$ on the compactification $\mathbb{R} \subset \mathbb{R} \cup\{\infty\}=\mathbb{R} \mathbb{P}^{1} \sim \mathbb{S}^{1}$. We use in general $E=F=\{0, \ldots, n-1\}$ or $E=F=\{1, \ldots, n\}$ for spindles with $n$ lines and suitable subsets for subspindles. For $e \in E$ we denote by $(e, \sigma(e))$ the corresponding line of the spindle configuration associated in the obvious way to $\sigma$ and we identify $\sigma$ with the set $\left\{(e, \sigma(e)\}_{e \in E}\right.$ of its lines. For $e \in E$, the notation $\sigma \backslash(e, \sigma(e))$ denotes the spindle or spindle-permutation obtained by restricting $\sigma$ to $E \backslash\{e\}$.

For $e_{1}<e_{2} \in E$ and $f_{1}<f_{2} \in F$ we denote by $\left[e_{1}, e_{2}\right]$, respectively $\left[f_{1}, f_{2}\right]$, the subset $\left(\left[e_{1}, e_{2}\right] \cap E\right) \subset E$, respectively $\left(\left[f_{1}, f_{2}\right] \cap F\right) \subset F$. For subsets $E^{\prime} \subset E, F^{\prime} \subset F$ of the same cardinality such that $\sigma(e) \in F^{\prime}$ for all $e \in E^{\prime}$ we denote by $\left(E^{\prime}, \sigma\left(E^{\prime}\right)=F^{\prime}\right)$ the spindle-permutation obtained by restricting $\sigma$ to $E^{\prime}$. A subblock of $\sigma$ can thus be written as $\left(\left[e_{1}, e_{2}\right], \sigma\left(\left[e_{1}, e_{2}\right]\right)\right) \subset \sigma$ or $\left(\left[e_{1}, e_{2}\right],\left[f_{1}, f_{2}\right]\right) \subset \sigma$. Sometimes we will also use the notation $\sigma\left(\left[e_{1}, e_{2}\right]\right)=\left[f_{1}, f_{2}\right]$. In the sequel, a $k$-block (of a spindlepermutation $\sigma$ ) will almost always denote a cyclic block consisting of $k$ lines, that is a subset $E^{\prime} \subset E$ of $k$ cyclically consecutive elements with $\sigma\left(E^{\prime}\right)$ cyclically consecutive in $F$.

Notice that given a spindle-permutation $\sigma: E \longrightarrow F$, its mirror configuration is for instance associated to the spindle-permutation $\bar{\sigma}: E \longrightarrow \bar{F}$ where $\bar{F}=F$ as a set but equipped with the opposite (cyclic) order. The application $\sigma \longmapsto \bar{\sigma}$ which replaces a spindle-permutation by its mirror enjoys good properties (preserves the spindleequivalence relation, the isotopy relation, yields a bijection between subblocks, etc) and will often be used for reducing the number of possible cases. 
The proof of Theorem 6.4 is by induction on the number of lines involved in $\sigma$ and $\sigma^{\prime}$. The result holds clearly for configurations with less than 4 lines (in this case, spindle-moves generate the complete permutation group of all lines).

Call a permutation irreducible if it contains no non-trivial block (consisting of $2 \leq k \leq$ $n-2$ cyclically consecutive lines).

Call a block minimal if it consists of $2 \leq k \leq n-2$ lines and if it contains no subblock of strictly smaller cardinality $k^{\prime} \geq 2$.

Proposition 6.1 shows that the set of possible subblocks of a spindle-permutation $\sigma$ is encoded by its linking matrix. Thus, either both or none of the spindle permutations $\sigma, \sigma^{\prime}$ are irreducible.

The proof of Theorem 6.4 splits into two cases, depending on the reducibility of $\sigma$ and $\sigma^{\prime}$.

\subsection{The reducible case}

Consider a non-trivial $k$-block $B \subset \sigma$. Corollary 6.2 shows that, up to spindle moves, $B^{\prime}=\iota(B)$ is a non-trivial $k$-block of $\sigma^{\prime}$.

Up to circular moves, we can assume that $B$ and $B^{\prime}$ are given by $\sigma([1, k])=[1, k]$ and $\sigma^{\prime}([1, k])=[1, k]$. We denote by $\bar{B}=\sigma \backslash B$ and $\bar{B}^{\prime}=\sigma^{\prime} \backslash B^{\prime}$ the complementary blocks.

By induction on the number of lines, the bijection of lines obtained by restricting $\iota$ to the subspindles $(1, \sigma(1)) \cup \bar{B}$ and $\left(\iota(1), \sigma^{\prime}(\iota(1))\right) \cup \overline{B^{\prime}}$ can be obtained by an isotopy $\mu$ which is a composition of spindle-moves. Up to a vertical reflection of $B$, the isotopy $\mu$ can be extended uniquely to all lines of $\sigma$ by considering the block $B \subset \sigma$ (represented by the line $(1, \sigma(1)))$ as rigid. We allow thus only spindle moves having the same effect on all lines of $B$. Replacing $\iota$ with the isotopy $\mu^{-1} \circ \iota$ we can suppose that the permutation induced by $\iota$ fixes all lines of $\bar{B}=\sigma \backslash B$. Applying the above argument to the complementary block $\bar{B}$ and working with $B \cup(k+1, \sigma(k+1))$, we get (after gluing back the block $\bar{B}$ onto the line $(k+1, \sigma(k+1)))$ an isotopy that consists only of spindle-moves, transforms $\sigma$ into $\sigma^{\prime}$ (up to a vertical and/or horizontal reflection of the block $\bar{B}$ ) and induces the line-bijection prescribed by the initial isotopy $\iota$.

\subsection{The irreducible case}

The case where $\sigma$ and $\sigma^{\prime}$ are irreducible is more complicated. It splits into the following three subcases: 
Subcase (1) There exists a line $L \in \sigma$ with $\sigma \backslash L$ irreducible.

Subcase (2) There exists a line $L$ such that $\sigma \backslash L$ contains a minimal block $B$ consisting of $3 \leq k \leq n-4$ lines.

Subcase (3) For every line $L \in \sigma$, the subspindle $\sigma \backslash L$ contains a 2-block.

Subcases (1) and (2) are dealt with by induction on the number of lines. A spindlepermutation in Subcase (3) is called exceptional. Subcase (3) is handled by classifying all exceptional spindle-permutations.

6.2.1 Subcase (1) Choose a line $L \in \sigma$ with $\sigma \backslash L$ irreducible. By Corollary 6.2, the spindle-permutation $\sigma^{\prime} \backslash \iota(L)$ is also irreducible. Up to circular moves of $\sigma$ and $\sigma^{\prime}$ we can assume $L=(n, n) \in \sigma, \iota(L)=(n, n) \in \sigma^{\prime}$.

By induction on $n$, the restriction of $\iota$ to the spindle configurations $\sigma([1, n-1])=$ $[1, n-1], \sigma^{\prime}([1, n-1])=[1, n-1]$ yields a bijection between their lines which can be realized by spindle moves. By irreducibility of $\sigma \backslash L$ and $\sigma^{\prime} \backslash \iota(L)$ and by induction on the number of lines, there exist (up to horizontal and vertical reflections of $\sigma^{\prime}$ ) integers $0 \leq \alpha, \beta<n-1$ such that

$$
\sigma^{\prime}(i)=\sigma(i-\alpha \quad(\bmod (n-1)))+\beta \quad(\bmod (n-1))
$$

for $i=1, \ldots, n-1$ where $x(\bmod (n-1)) \in\{1, \ldots, n-1\}$. Thus a line $(i, \sigma(i))=$ $(i, j)$ (with $1 \leq i \leq n-1)$ of $\sigma$ corresponds to the line $(i+\alpha, j+\beta)$ of $\sigma^{\prime}$ (where the numbers $i+\alpha, j+\beta \in\{1, \ldots, n-1\}$ are modulo $n-1)$. We say that a line $(i, \sigma(i))$ (with $i<n$ ) is moved through infinity if either $i \geq n-\alpha$ or $\sigma(i) \geq n-\beta$. If both inequalities hold, we say that $(i, \sigma(i))$ is moved twice through infinity.

If there exists a line $(i, \sigma(i))$ which is moved exactly once through infinity, then every line $(j, \sigma(j)), 1 \leq j \leq n-1$ is moved exactly once through infinity: Otherwise, consider a line $(i, \sigma(i))$ which is moved once and a line $(j, \sigma(j))$ which is not moved through infinity or moved twice. This implies that the 3 -subspindles

$$
\{(i, \sigma(i)),(j, \sigma(j)),(n, n)\} \text { and }\{\iota(i, \sigma(i)), \iota(j, \sigma(j)), \iota(n, n)=(n, n)\}
$$

are mirrors (and thus of different vorticities and not isotopic) which is impossible. Thus every line of $\sigma \backslash(n, n)$ is moved through infinity exactly once which implies $\alpha+\beta=n-1$ and $\sigma([1, \beta])=[n-\beta, n-1], \sigma([\beta+1, n-1])=\sigma([n-\alpha, n-1])=[1, \alpha]$. This contradicts the irreducibility of $\sigma$.

We can now assume that every line of $\sigma \backslash(n, n)$ is moved an even number of times through infinity. This implies $\alpha=\beta$ and the existence of a non-trivial subblock of the form $\sigma([n-\alpha, n-1])=[n-\alpha, n-1]$ or $\sigma([n-\alpha, n])=[n-\alpha, n]$ in $\sigma$ for $\alpha>0$. We have thus $\alpha=\beta=0$ which shows $\sigma=\sigma^{\prime}$ and proves the result. 
6.2.2 Subcase (2) Up to circular moves, we can assume that $\sigma \backslash(n, n)$ contains a minimal block $B$ of size $3 \leq \sharp(B) \leq n-4$. Irreducibility of $\sigma$ shows the existence (up to horizontal and vertical reflections of $\sigma$ ) of integers $\alpha, \beta \geq 1$ with $3 \leq \alpha+\beta=$ $\sharp(B) \leq n-4$ and of an integer $\gamma$ with $1 \leq \gamma<n-1-\alpha-\beta$ such that $B$ is given by

$$
\sigma([1, \alpha] \cup[n-\beta, n-1])=[\gamma+1, \gamma+\alpha+\beta],
$$

see Figure 9 with $B$ included in the shaded region. Up to considering mirror-configurations, we can suppose $\gamma<\sigma(\alpha)<\sigma(n-\beta) \leq \alpha+\beta+\gamma$. There exist now indices $v_{1}, v_{2}$ such that $\alpha<v_{1}<v_{2}<n-\beta$ and $\sigma\left(v_{1}\right)>\alpha+\beta+\gamma, \sigma\left(v_{2}\right) \leq \gamma$ (see Figure 9). Indeed, otherwise $\sigma([\alpha+1, \alpha+\gamma])=[1, \gamma]$ and $\sigma([\alpha+\gamma+1, n-\beta-1])=$ $[\alpha+\beta+\gamma+1, n-1]$ which implies the existence of a non-trivial subblock in $\sigma$ and contradicts the irreducibility of $\sigma$.

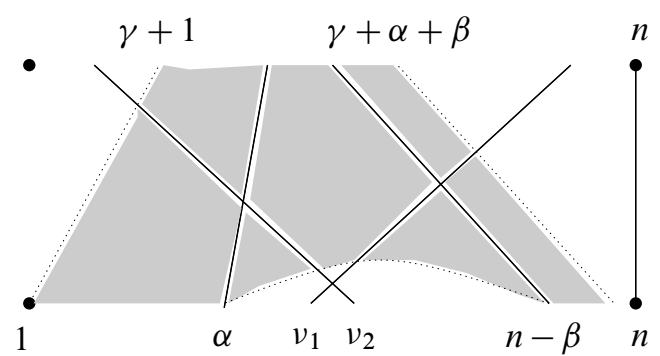

Figure 9: A schematical picture of the subspindle $\widetilde{\sigma} \subset \sigma$.

Lemma 6.6 The subspindle $\tilde{\sigma} \subset \sigma$ defined by

$$
B \cup\left(v_{1}, \sigma\left(v_{1}\right)\right) \cup\left(v_{2}, \sigma\left(v_{2}\right)\right) \cup(n, n)
$$

is irreducible.

Proof Consider the 5-subspindle $\tau \subset \widetilde{\sigma}$ containing the five lines

$$
(\alpha, \sigma(\alpha)),\left(v_{1}, \sigma\left(v_{1}\right)\right),\left(v_{2}, \sigma\left(v_{2}\right)\right),(n-\beta, \sigma(n-\beta)),(n, n) .
$$

The inequalities $\alpha<v_{1}<v_{2}<n-\beta<n$ and $\sigma\left(v_{2}\right)<\sigma(\alpha)<\sigma(n-\beta)<\sigma\left(v_{1}\right)<n$ imply easily that $\tau$ is irreducible (it is enough to check that $\tau$ contains no 2-block). Thus any block $\widetilde{B} \subset \widetilde{\sigma}$ intersects $\tau$ in a subset with cardinality $\sharp(\widetilde{B} \cap \tau) \in\{0,1,4,5\}$. Up to replacing $\widetilde{B}$ by its complementary block $\widetilde{\sigma} \backslash \widetilde{B} \subset \widetilde{\sigma}$, we can assume $\sharp(\widetilde{B} \cap \tau) \leq 1$. If $\widetilde{B} \cap \tau=(\alpha, \sigma(\alpha))$ or $\widetilde{B} \cap \tau=(n-\beta, \sigma(n-\beta))$, then $\widetilde{B} \subset B$ is also a non-trivial subblock in $\sigma$. This contradicts the irreducibility of $\sigma$. 
If $\widetilde{B} \cap \tau=\{(n, n)\}$, then a non-trivial subblock $\widetilde{B}$ of $\widetilde{\sigma}$ contains also at least one line of the set $\left\{\left(v_{1}, \sigma\left(v_{1}\right)\right),\left(v_{2}, \sigma\left(v_{2}\right)\right)\right\}$. This contradicts our assumption $\sharp(\widetilde{B} \cap \tau) \leq 1$.

If $\widetilde{B} \cap \tau=\left\{\left(v_{1}, \sigma\left(v_{1}\right)\right)\right\}$, then a non-trivial subblock $\widetilde{B}$ contains also at least one line of $\left\{(\alpha, \sigma(\alpha)),\left(v_{2}, \sigma\left(v_{2}\right)\right)\right\}$, contradicting again $\sharp(\widetilde{B} \cap \tau) \leq 1$.

The case $\widetilde{B} \cap \tau=\left\{\left(v_{2}, \sigma\left(v_{2}\right)\right)\right\}$ is analogous.

The case $\widetilde{B} \cap \tau=\varnothing$ implies that $\widetilde{B}$ is a block of $\sigma$. Since $\sigma$ is irreducible, this is impossible.

Apply now Theorem 6.4 to $\iota$ restricted to the subspindles $\widetilde{\sigma}$ and $\tilde{\sigma}^{\prime}=\iota(\tilde{\sigma}) \subset \sigma^{\prime}$ (which contain at most $n-1$ lines). By minimality of $B$, the subset $B^{\prime}=\iota(B)$ is already a subblock in $\sigma^{\prime} \backslash \iota(n, n)$, see Remark 6.3. Up to spindle moves, the relative position of the subblock $B^{\prime} \subset \sigma^{\prime} \backslash(n, n)$ (with $\left.(n, n)=\iota(n, n)\right)$ inside $\sigma^{\prime}$ is described by integers $\alpha^{\prime}, \beta^{\prime}, \gamma^{\prime}$ defined in the obvious way. Applying Theorem 6.4 to the restriction of $\iota$ to the irreducible subspindle $\tilde{\sigma}$ we get $\alpha^{\prime}=\alpha, \beta^{\prime}=\beta$. The number $\gamma$, respectively $\gamma^{\prime}$, is given by the number of triplets $\{(1, \sigma(1)),(j, \sigma(j)),(n, n)\}$, respectively $\left\{\left(1, \sigma^{\prime}(1)\right),\left(j, \sigma^{\prime}(j)\right),(n, n)\right\}$ of vorticity -1 (isotopic to $\left.\{(1,2),(2,1),(3,3)\}\right)$ with $\alpha=\alpha^{\prime}<j<n-\beta=n-\beta^{\prime}$. These numbers can be retrieved from the linking matrix of $\sigma$, respectively $\sigma^{\prime}$, and we have thus $\gamma=\gamma^{\prime}$. This implies that the permutation induced by $\iota$ fixes all lines of $B$ and $B=B^{\prime}$ is thus a common subspindle of $\sigma$ and $\sigma^{\prime}$.

Denoting by $\bar{B}=\sigma \backslash(B \cup(n, n))$ the complementary subblock of $B$ in $\sigma \backslash(n, n)$, the above discussion shows that $\iota(\bar{B})$ is a block in $\sigma^{\prime} \backslash(n, n)$. Since the proof of Lemma 6.6 does not request minimality, we can find an irreducible subspindle $\bar{\sigma}$ containing $\bar{B},(n, n)$ and two suitable lines of $B$. Applying Theorem 6.4 inductively to the restriction of $\iota$ to the subspindles $\bar{\sigma} \subset \sigma$ and $\bar{\sigma}^{\prime}=\iota(\bar{\sigma}) \subset \sigma^{\prime}$ (containing by assumption at most $n-1$ lines) shows that the permutation induced by $\iota$ fixes also the set $\bar{B}$. This implies $\sigma=\sigma^{\prime}$ and proves Subcase (2).

6.2.3 Exceptional irreducible spindles Call a spindle-permutation $\sigma$ of $n \geq 4$ lines exceptional if $\sigma$ is irreducible and $\sigma \backslash L$ contains a (cyclic) 2-block for every line $L \in \sigma$.

\section{Proposition 6.7}

(i) For $n \geq 5$ odd, the spindle-permutation $\tau=\tau_{n}$ of $\{0, \ldots, n-1\}$ defined by

$$
\tau: i \longmapsto \tau(i)=2 i \quad(\bmod n), 0 \leq i \leq n-1
$$


and its mirror $\bar{\tau}=\bar{\tau}_{n}$ given (up to circular moves) by

$$
\bar{\tau}: i \longmapsto \tau(i)=-2 i \quad(\bmod n), 0 \leq i \leq n-1
$$

are exceptional. The spindle-permutations $\tau_{5}$ and $\bar{\tau}_{5}$ are spindle-equivalent. For $n>5$ odd, the spindle-permutations $\tau_{n}$ and $\bar{\tau}_{n}$ are not spindle-equivalent and have linking matrices in different switching classes. In particular, their associated spindle configurations are not isotopic.

(ii) The spindle-permutations $\tau_{n}, \bar{\tau}_{n}, n \geq 5$ odd, are the only exceptional spindlepermutations having more than 3 lines, up to spindle-equivalence.

(iii) If $\iota$ is an isotopy of the exceptional spindle configuration $\tau_{n}$ onto itself, then the line permutation $L \longmapsto \iota(L)$ induced by $\iota$ can be realized by spindle moves.

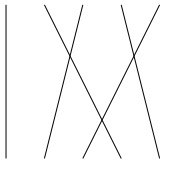

$\tau_{5}$

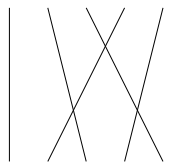

$\bar{\tau}_{5}$

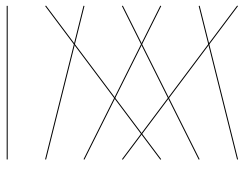

$\tau_{7}$

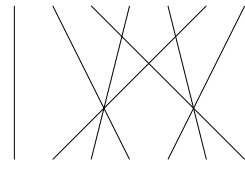

$\bar{\tau}_{7}$

Figure 10: The exceptional spindles $\tau_{5}, \bar{\tau}_{5}, \tau_{7}, \bar{\tau}_{7}$.

Proof We write $\tau=\tau_{n}$ for $n \geq 5$ odd. We have $\tau=\tilde{\tau}^{k}$ with

$$
\tilde{\tau}^{k}: i \longmapsto \tilde{\tau}^{k}(i)=2 k+\tau(i-k \quad(\bmod n)) \quad(\bmod n)
$$

showing that $\tau$ has a group of automorphisms acting transitively on its lines. Since $\tau \backslash(0,0)$ contains the (cyclic) 2 -block $\left\{\left(\frac{n-1}{2}, n-1\right),\left(\frac{n+1}{2}, 1\right)\right\}$, we get thus a cyclic 2-block in $\tau \backslash L$ for any line $L \in \tau$.

Suppose now that $\tau$ is reducible and consider a non-trivial subblock $B \subset \tau$. Up to replacing $B$ by the complementary block $\tau \backslash B$, we can assume that $B$ contains fewer than $\frac{n}{2}$ lines. Up to cyclic moves we can assume that $(0,0),(1,2) \in B$. This implies either $B=\tau \backslash\left(\frac{n+1}{2}, 1\right)$ which contradicts the non-triviality of $B$ or $B$ contains the line $\left(\frac{n+1}{2}, 1\right)$. But then $B$ contains either all lines $(k, 2 k), 1 \leq k \leq \frac{n-1}{2}$ or all lines $(k, 2 k-n), \frac{n+1}{2} \leq k \leq n-1$. Since $(0,0) \in B$, we have in both cases $\sharp(B) \geq \frac{n+1}{2}$ which contradicts the assumption $\sharp(B)<\frac{n}{2}$.

This shows that $\tau=\tau_{n}$ and its mirror $\bar{\tau}_{n}$ are exceptional.

A horizontal reflection transforms $\tau_{5}$ into $\bar{\tau}_{5}$ which proves their equivalence under spindle moves (see Figure 10). 
For $n>5$ odd, $\tau_{n} \backslash L$ and $\bar{\tau}_{n} \backslash \bar{L}$ contain both a unique 2-block $B$, resp. $\bar{B}$ (the choice of the lines $L, \bar{L}$ is irrelevant since they are transitively permuted by automorphisms). We get 3-subspindles $L \cup B \subset \tau_{n}$ and $\bar{L} \cup \bar{B} \subset \bar{\tau}_{n}$ which are not isotopic since they are mirrors and have opposite vorticities (see Figure 10). Since such a 3 -spindle and its isotopy class can be recovered from the linking matrix, the associated linking matrices are not switching equivalent and the corresponding spindle configurations are not isotopic. This proves assertion (i).

(Note that this argument fails for $n=5$ : In this case, $\tau \backslash L$ gives rise to two complementary (cyclic) 2-blocks $B, \bar{B}$ such that $L \cup B$ and $L \cup \bar{B}$ are non-isotopic 3-spindles.)

An inspection shows that no irreducible 4-spindle exists. This proves assertion (ii) for $n=4$. Hence, we can suppose $n \geq 5$.

Consider an exceptional spindle permutation $\sigma$ on $n \geq 5$ lines. For each line $L$, choose a 2-block $B_{L} \subset \sigma \backslash L$. We call the set $B_{L} \subset \sigma$ a line-block. The condition $n \geq 5$ ensures that we have exactly $n$ different line-blocks.

Suppose first the existence of a line $(a, b) \in B_{L_{1}} \cap B_{L_{2}} \cap B_{L_{3}}$ which is common to three different line-blocks. Up to replacing $\sigma$ by its mirror-permutation, circular moves, vertical and horizontal reflections, we can assume $(a, b)=(2,2)$ and $B_{L_{1}} \cup$ $B_{L_{2}} \cup B_{L_{3}}=\{(0, c),(1,0),(2,2),(3,4)\}$ with $c \in\{1,3\}$. If $c=1$, the existence of the 2-block $\{(0,1),(1,0)\}$ contradicts the irreducibility of $\sigma$. If $c=3$ (see Figure 11), the line-block $B_{(2,2)}$ defined by $(2,2)$ is either $\{(1,0),(3,4)\}$ which implies $n=5$ and $\sigma=\tau_{5}$ or $\{(0,3),(n-1,1)\}$. If $n=5$ we get $\sigma=\tau_{5}$. If $n>5$, the existence of the non-trivial sub-block $\{(0,3),(1,0),(2,2),(n-1,1)\}$ contradicts the irreducibility of $\sigma$. Hence a line $(a, b)$ is included in at most two line-blocks.

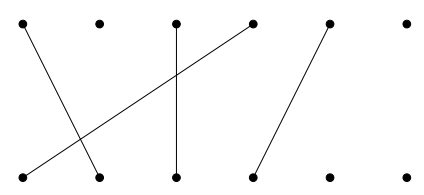

Figure 11: $c=3$ for three intersecting line-blocks.

Since every line-block $B_{L}$ in $\sigma$ contains two lines of $\sigma$, we can now assume that every line of $\sigma$ is contained in exactly two different line-blocks $B_{L_{1}}, B_{L_{2}}$.

Consider two graphs $\Gamma_{\sigma}^{0}, \Gamma_{\sigma}^{1}$ with vertices $\{0,1, \ldots, n-1\}$ and edges defined as follows: An edge $\{i, j\}$ of $\Gamma_{\sigma}^{0}$ (respectively $\{\sigma(i), \sigma(j)\}$ of $\left.\Gamma_{\sigma}^{1}\right)$ exists if $\{(i, \sigma(i)),(j, \sigma(j))\}$ is a line-block of $\sigma$. Such an edge $\{i, j\} \subset \Gamma_{\sigma}^{0}$ (respectively $\{\sigma(i), \sigma(j)\} \subset \Gamma_{\sigma}^{1}$ ) is of type $k \geq 1$ if $j=i \pm k(\bmod n)(\operatorname{respectively} \sigma(j)=\sigma(i) \pm k(\bmod n))$. The 
definition of an edge implies $k \in\{1,2\}$ and the application $i \longmapsto \sigma(i)$ induces an isomorphism from $\Gamma_{\sigma}^{0}$ onto $\Gamma_{\sigma}^{1}$ sending an edge of type $k$ in $\Gamma_{\sigma}^{0}$ onto an edge of type $3-k$ in $\Gamma_{\sigma}^{1}$. Our assumptions imply that $\Gamma_{\sigma}^{0}$ and $\Gamma_{\sigma}^{1}$ are 2 -regular graphs with connected components given by cyclic graphs containing at least 3 vertices.

Suppose first that (a connected component of) $\Gamma_{\sigma}^{0}$ contains two adjacent edges of different types. Up to a vertical reflection and circular moves, we can assume that these edges are either $\{1,2\},\{1,3\}$ or $\{1,2\},\{2,4\}$.

If $\Gamma_{\sigma}^{0}$ contains the edges $\{1,2\},\{1,3\}$, we can assume, up to replacing $\sigma$ by its mirror permutation and circular moves, that $\sigma$ contains the lines $(1,2),(2,4)$ and $(3, c)$ with $c \in\{1,3\}$. The case $c=3$ leads to a 2 -block $\{(2,4),(3,3)\}$ in $\sigma$ which is impossible. $\sigma$ contains thus the lines $(1,2),(2,4),(3,1)$ (see Figure 12).

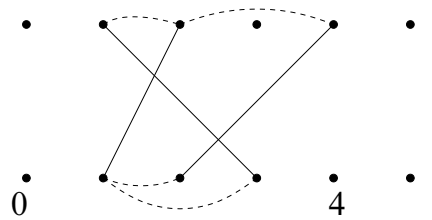

Figure 12: The case $c=3$, together with parts of $\Gamma_{\sigma}^{0}$ (dashed lines at the bottom) and $\Gamma_{\sigma}^{1}$ (dashed lines at the top).

Consider now the vertex 3 of degree 2 in the graph $\Gamma_{\sigma}^{1}$. Since the vertex 2 is already involved in the edges $\{1,2\}$ (corresponding to the line-block $B_{(2,4)}=\{(1,2),(3,1)\} \subset$ $\sigma \backslash(2,4))$ and $\{2,4\}$ (corresponding to the line-block $B_{\left(\sigma^{-1}(3), 3\right)}=\{(1,2),(2,4)\} \subset \sigma \backslash$ $\left.\left(\sigma^{-1}(3), 3\right)\right)$ of $\Gamma_{\sigma}^{1}$, the vertex 3 of $\Gamma_{\sigma}^{1}$ has its two neighbours in the set $\{1,4,5\}$ and is either adjacent to vertex 1 or to vertex 4 (and perhaps to both). In the first case, the lineblock corresponding to the edge $\{1,3\}$ of $\Gamma_{\sigma}^{1}$ must be $B_{(1,2)}=\left\{(3,1),\left(\sigma^{-1}(3), 3\right)\right\}=$ $\{(3,1),(4,3)\}$ and we get the existence of the lines $(1,2),(2,4),(3,1),(4,3)$ in $\sigma$ which implies $\sigma=\tau_{5}$ if $n=5$ and the existence of a non-trivial subblock $\{(1,2)$, $(2,4),(3,1),(4,3)\}$ for $n>5$. In the second case, the existence of the edge $\{3,4\}$ in $\Gamma_{\sigma}^{1}$ implies the existence of a line $(c, 3)$ with $c \in\{0,4\}$ in $\sigma$. The case $c=0$ creates a 2 -block $\{(0,3),(1,2)\}$ in $\sigma$ and is excluded. The case $c=4$ leads to $\sigma=\tau_{5}$ for $n=5$ or to the existence of the non-trivial subblock $\{(1,2),(2,4),(3,1),(4,3)\}$ for $n>5$.

Consider now the case where $\Gamma_{\sigma}^{0}$ contains the adjacent edges $\{1,2\}$ and $\{2,4\}$ of different types. Since the vertex 2 of $\Gamma_{\sigma}^{0}$ has already two neighbours 1 and 4 , the arguments considered a few lines above apply and the vertex 3 is either adjacent to 1 or to 4 (and perhaps to both). The first case, where $\Gamma_{\sigma}^{0}$ contains the edges $\{1,2\}$ and 
$\{1,3\}$, has already been discussed. The second one can be reduced to the first case after a vertical reflection and a circular move.

We can thus suppose that all edges of a connected component in $\Gamma_{\sigma}^{0}$ are of the same type which can be assumed to be 1, up to a horizontal reflection. This shows, that $\sigma$ consists (up to circular moves) of the lines

$$
(0,0),(1, \pm 2),(2, \pm 4), \ldots,(k, \pm 2 k), \ldots,(n-1, \pm 2(n-1))
$$

which implies $n$ odd and $\sigma=\tau_{n}$ or $\sigma=\bar{\tau}_{n}$ and proves assertion (ii).

Up to considering mirror configurations, it is enough to prove assertion (iii) for $\tau_{n}$. We consider first the case $n=5$. Up to circular moves, vertical and horizontal reflections, we can assume that an isotopy $\iota: \tau_{5} \longrightarrow \tau_{5}$ induces the identity-permutation on the lines $(0,0)$ and $(1,2)$. An inspection of isotopy types of triplets $\{(0,0),(1,2),(i, 2 i$ $(\bmod 5))\}$ shows that $\iota(3,1)=(3,1)$. A similar argument yields then $\iota(2,4)=(2,4)$ and completes the proof for $\tau_{5}$.

We consider now an isotopy of $\sigma=\tau_{n}$ for an odd integer $n>5$. The definition of the graph $\Gamma_{\sigma}^{0}$ (see the proof of assertion (ii) above) with vertices $\{0, \ldots, n-1\}$ and edges $\{i, i+1(\bmod n)\}$ corresponding to line-blocks $B_{\left(\frac{2 i+1}{2}(\bmod n), 2 i+1\right)}=\{(i, 2 i$ $(\bmod n)),(i+1,2 i+2(\bmod n))\}$ implies that $\iota$ induces a graph automorphism of $\Gamma_{\sigma}^{0}$. Up to circular moves and a vertical reflection, we can thus assume that $\iota$ induces the identity automorphism of $\Gamma_{\sigma}^{0}$ and thus the identity permutation on the lines of $\sigma=\tau_{n}$.

Proof of Theorem 6.4 Follows from Section 6.1 and Section 6.2

\section{Theorem 3.2 of Kashin-Mazurovskiǐ}

In our terminology, [7, Theorem 3.2] can be restated as follows:

Theorem 7.1 Spindle configurations with switching-equivalent linking-matrices are isotopic.

We prove it by constructing an isotopy between the two spindle configurations.

Let $\sigma$ and $\mu$ be two spindles with switching-equivalent linking matrices $X_{\mu}=P^{t} X_{\sigma} P$ where $P$ is a signed permutation matrix inducing a bijection between the lines of $\sigma$ and $\mu$. (Recall that the linking matrix $X_{\tau}$ of a spindle with permutation $\tau$ is defined by

$$
\left.\left(X_{\tau}\right)_{i, j}=\operatorname{sign}((i-j)(\tau(i)-\tau(j))) .\right)
$$


Up to circular moves, we can assume that the line-bijection

$$
(i, \sigma(i)) \longmapsto\left(i^{\prime}, \mu\left(i^{\prime}\right)\right)
$$

induced by $P$ sends the first line $(1, \sigma(1)=1)$ of $\sigma$ onto the first line $(1, \mu(1)=1)$ of $\mu$. This implies that the conjugating matrix $P$ is an ordinary permutation matrix and thus we have

$$
(i-j)(\sigma(i)-\sigma(j))\left(i^{\prime}-j^{\prime}\right)\left(\mu\left(i^{\prime}\right)-\mu\left(j^{\prime}\right)\right)>0
$$

for all $1 \leq i<j \leq n$.

For $t \in \mathbb{R}$ fixed, consider the line $L_{i}(t)=s P_{\omega}^{i}(t)+(1-s) P_{\alpha}^{i}(t)$ parametrized by $s \in \mathbb{R}$, oriented from $P_{\alpha}^{i}(t)$ to $P_{\omega}^{i}(t)$ where

$$
\left\{\begin{array}{l}
P_{\alpha}^{i}(t)=(1-t)(i, 1,0)+t\left(0,1, i^{\prime}\right) \\
P_{\omega}^{i}(t)=(1-t)(0,-1, \sigma(i))+t\left(-\mu\left(i^{\prime}\right),-1,0\right) .
\end{array}\right.
$$

Proposition 7.2 For $t \in \mathbb{R}$, the lines $\mathcal{L}(t)=\left\{L_{1}(t), \ldots, L_{n}(t)\right\}$ form a skew configuration.

Moreover, $\mathcal{L}(0)$ realizes the spindle $\sigma$ and $\mathcal{L}(1)$ realizes the spindle $\mu$.

This is essentially [7, Theorem 3.2]. The following proof is a transcription of the original proof in [7], made slightly more elementary in the sense that we avoid using [7, Theorem 2.13] (involving configurations of subspaces in spaces of dimension higher than 3 ) at the cost of a determinant-computation.

Proof We have to show that

$$
\operatorname{lk}\left(L_{i}(t), L_{j}(t)\right)=\operatorname{sign}\left(\begin{array}{c}
P_{\omega}^{i}(t)-P_{\alpha}^{i}(t) \\
P_{\alpha}^{j}(t)-P_{\omega}^{i}(t) \\
P_{\omega}^{j}(t)-P_{\alpha}^{j}(t)
\end{array}\right)
$$

is constant. The matrix is given by

$$
\left(\begin{array}{ccc}
(t-1) i-t \mu\left(i^{\prime}\right) & -2 & (1-t) \sigma(i)-t i^{\prime} \\
(1-t) j+t \mu\left(i^{\prime}\right) & 2 & (t-1) \sigma(i)+t j^{\prime} \\
(t-1) j-t \mu\left(j^{\prime}\right) & -2 & (1-t) \sigma(j)-t j^{\prime}
\end{array}\right)
$$

and its determinant $p$ equals

$$
\begin{gathered}
p=2\left((i-j)(\sigma(i)-\sigma(j))+\left(i^{\prime}-j^{\prime}\right)\left(\mu\left(i^{\prime}\right)-\mu\left(j^{\prime}\right)\right)\right) t^{2} \\
-4(i-j)(\sigma(i)-\sigma(j)) t+2(i-j)(\sigma(i)-\sigma(j)) .
\end{gathered}
$$


The discriminant (with respect to $t$ ) of $p$ given by

$$
-16(i-j)(\sigma(i)-\sigma(j))\left(i^{\prime}-j^{\prime}\right)\left(\mu\left(i^{\prime}\right)-\mu\left(j^{\prime}\right)\right)
$$

is strictly negative for $i \neq j$ which shows that $p$ is non-zero for all $t \in \mathbb{R}$. This proves that $\mathcal{L}(t)$ is a skew configuration for all $t \in \mathbb{R}$.

For $t=0$, we get a spindle with axis $(\mathbb{R}, 1,0)$ and directrix $(0,-1, \mathbb{R})$ realizing the spindle $\sigma$ with lines

$$
L_{i}=s(i, 1,0)+(1-s)(0,-1, \sigma(i)), i=1, \ldots, n, s \in \mathbb{R} .
$$

For $t=1$, we get a spindle with axis $(0,1, \mathbb{R})$ and directrix $(-\mathbb{R},-1,0)$ (where $-\mathbb{R}$ denotes the real line endowed with the opposite order) realizing $\mu$ with lines

$$
L_{i^{\prime}}^{\prime}=s\left(0,1, i^{\prime}\right)+(1-s)\left(-\mu\left(i^{\prime}\right),-1,0\right), i^{\prime}=1, \ldots, n, s \in \mathbb{R} .
$$

The proof of Theorem 7.1 is immediate.

Proof of Corollary 1.3 Spindle-equivalent permutations give rise to isotopic spindles and their linking matrices are thus switching-equivalent.

On the other hand, given two permutations $\sigma, \sigma^{\prime}$ with switching-equivalent linking matrices, Theorem 7.1 yields an isotopy between the associated spindle configurations and Theorem 1.1 implies that $\sigma, \sigma^{\prime}$ are spindle-equivalent.

\section{Spindlegenus}

We describe a topological invariant of permutations up to spindle-equivalence. This yields an invariant for spindle configurations by Theorem 1.1.

Let $P$ be a regular polygon with $n$ edges $E_{1}, \ldots, E_{n}$ in clockwise cyclical order. Reading indices modulo $n$ we orient the edge $E_{i}$ from $E_{i-1} \cap E_{i}$ to $E_{i} \cap E_{i+1}$ and denote by $E_{-i}$ the edge $E_{i}$ with the opposite orientation. Consider a second polygon $P^{\prime}$ with edges $E_{1}^{\prime}, \ldots, E_{n}^{\prime}$ obtained from $P$ by an orientation-reversing isometry (for example an orthogonal symmetry with respect to a line). Given a permutation $\sigma:\{1, \ldots, n\} \longrightarrow\{1, \ldots, n\}$, gluing the oriented edge $E_{i} \in P$ onto the oriented edge $E_{\sigma(i)}^{\prime} \in P^{\prime}$ for $1 \leq i \leq n$ yields a compact orientable surface $\Sigma(\sigma)$. We call the genus $g(\sigma) \in \mathbb{N}$ of $\Sigma(\sigma)$ the spindlegenus of $\sigma$.

This construction can be generalized as follows:

A signed permutation is a permutation of the set $\{ \pm 1, \ldots, \pm n\}$ such that $\widetilde{\sigma}(-i)=$ $-\tilde{\sigma}(i)$. The group of all signed permutations is the full group of all isometries acting on 
the regular $n$-dimensional cube $[-1,1]^{n} \subset \mathbb{R}^{n}$. Such a permutation can be graphically presented by segments $[(i, 0),(|\widetilde{\sigma}(i)|, 1)]$ carrying signs $\epsilon_{i}=\frac{\widetilde{\sigma}(i)}{|\widetilde{\sigma}(i)|} \in\{ \pm 1\}$. The notion of spindle-equivalence extends to signed permutations in the obvious way. The construction of the compact surface $\Sigma(\sigma)$ can now be applied to a signed permutation $\tilde{\sigma}$ (glue the oriented edge $E_{i} \in P$ onto the oriented edge $E_{\tilde{\sigma}(i)}^{\prime} \in P^{\prime}$ for all $i=1, \ldots, n$ ) and yields a generally non-orientable surface $\Sigma(\widetilde{\sigma})$ presented as a $C W$-complex with two open 2-cells (corresponding to the interiors of the polygons $P$ and $P^{\prime}$ ), $n$ open 1 -cells (the edges of $P$ or $\left.P^{\prime}\right)$ and with $v(\widetilde{\sigma})$ points or 0 -cells. The surface $\Sigma(\widetilde{\sigma})$ is orientable if and only if $\left|\sum_{i=1}^{n} \epsilon_{i}\right|=n$, that is if $\widetilde{\sigma}$ is either an ordinary permutation or the opposite of an ordinary permutation. The Euler characteristic $2-n+v(\widetilde{\sigma})$ and orientability describe the surface $\Sigma(\widetilde{\sigma})$ up to homeomorphism (see for instance Massey [9]).

Proposition 8.1 If $\widetilde{\sigma}, \widetilde{\sigma}^{\prime}$ are two signed spindle-equivalent permutations, then $v(\widetilde{\sigma})=$ $v\left(\widetilde{\sigma}^{\prime}\right)$ and the compact surfaces $\Sigma(\widetilde{\sigma})$ and $\Sigma\left(\widetilde{\sigma}^{\prime}\right)$ are homeomorphic.

Corollary 8.2 Two permutations $\sigma$ and $\sigma^{\prime}$ which are spindle-equivalent have the same genus (that is $g(\sigma)=g\left(\sigma^{\prime}\right)$ ).

The following table lists the multiplicities for the spindlegenus $g(\sigma)$ (related to the number $v(\sigma)$ of vertices in the $C W$-complex considered above by the formula $\chi(\Sigma)=$ $2-n+v(\sigma)=2-2 g(\sigma)$ for the Euler characteristic of $\Sigma(\sigma))$ of permutations normalized by $\sigma(1)=1$. Multiplication by $n$ gives the corresponding numbers for all permutations:

\begin{tabular}{|c|c|c|c|c|c|c|}
\hline$n$ & $g=0$ & $g=1$ & $g=2$ & $g=3$ & $g=4$ & $g=5$ \\
\hline 1 & 1 & & & & & \\
2 & 1 & & & & & \\
3 & 1 & 1 & & & & \\
4 & 1 & 5 & & & & \\
5 & 1 & 15 & 8 & & & \\
6 & 1 & 35 & 84 & & & \\
7 & 1 & 70 & 469 & 180 & & \\
8 & 1 & 126 & 1869 & 3044 & & \\
9 & 1 & 210 & 5985 & 26060 & 8064 & \\
10 & 1 & 330 & 16401 & 152900 & 193248 & \\
11 & 1 & 495 & 39963 & 696905 & 2286636 & 604800 \\
12 & 1 & 715 & 88803 & 2641925 & 18128396 & 19056960 \\
\hline
\end{tabular}

(see also Sequence A60593 of [15]). 
Given a permutation $\sigma$ of $\{1, \ldots, n\}$ and a sequence of signs $\epsilon=\left(\epsilon_{1}, \ldots, \epsilon_{n}\right) \in\{ \pm 1\}^{n}$, we consider the signed permutation $\sigma_{\epsilon}: i \longmapsto \epsilon_{i} \sigma(i)$ and set

$$
V_{\sigma}(t, z)=\sum_{\left(\epsilon_{1}, \ldots, \epsilon_{n}\right) \in\{ \pm 1\}^{n}} z^{v\left(\sigma_{\epsilon}\right)} t^{\sum_{i} \epsilon_{i}} \in \mathbb{Z}\left[t, \frac{1}{t}, z\right] .
$$

Corollary 8.3 The application $\sigma \longmapsto V_{\sigma}(t, z)$ is well-defined on spindle-equivalence classes and satisfies

$$
V_{\bar{\sigma}}(t, z)=V_{\sigma}\left(\frac{1}{t}, z\right)
$$

where $\bar{\sigma}(i)=n+1-\sigma(i)$ is a mirror spindle-permutation of $\sigma$.

Proof of Proposition 8.1 The number $v(\widetilde{\sigma})$ is obviously invariant under circular moves.

The compact surface $\Sigma(\widetilde{\sigma})$ is orientable if and only if $\sum_{i=1}^{n} \epsilon_{i} \in\{ \pm n\}$ and $\sum_{i=1}^{n} \epsilon_{i}$ is preserved under spindle-equivalence. Hence it is enough to show the invariance under horizontal and vertical reflections of the number $v(\sigma)$ of vertices in the $C W$-complex representing $\Sigma(\widetilde{\sigma})$.

This number $v=v(\widetilde{\sigma})$ can be computed graphically as follows: Represent the signed permutation $\tilde{\sigma}(i)$ of $\sigma$ by drawing $n$ segments joining the points $(i, 0), i=1, \ldots, n$ to $(|\sigma(i)|, 1)$ as shown in Figure 13 for the signed permutation $\widetilde{\sigma}(1)=-2, \widetilde{\sigma}(2)=3$, $\tilde{\sigma}(3)=1$.

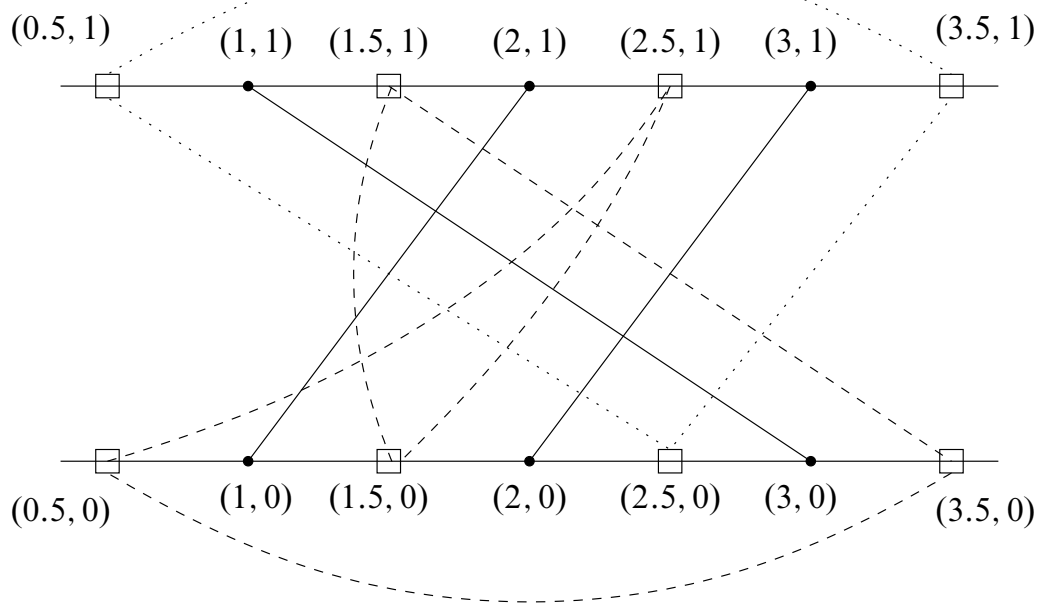

Figure 13: Example for the computation of $v(\widetilde{\sigma})$ 
Add $2 n+2$ additional points $(0.5,0),(1.5,0), \ldots,(n+0.5,0)$ and $(0.5,1),(1.5,1)$, $\ldots,(n+0.5,1)$ by drawing all points at height $y=0$ or $y=1$ which are at distance 0.5 from an endpoint of a segment $[(i, 0),(|\widetilde{\sigma}(i)|, 1)]$. Join $(0.5,0),(n+0.5,0)$ (respectively $(0.5,1),(n+0.5,1))$ by dotted arcs and join the points $(i \pm 0.5,0),(|\widetilde{\sigma}(i)| \pm$ $\left.\epsilon_{i} 0.5,1\right)$ by dotted arcs with $\epsilon_{i}=\frac{\tilde{\sigma}(i)}{|\tilde{\sigma}(i)|}$ corresponding to the sign of the $i$ th segment, see Figure 13 for an example. This yields a 2-regular graph with vertices

$$
(0.5,0),(1.5,0), \ldots,(n+0.5,0),(0.5,1),(1.5,1), \ldots,(n+0.5,1)
$$

by considering all dotted arcs as edges. In the example of Figure 13, this graph has two connected components with vertices

$$
(0.5,0),(3.5,0),(1.5,1),(1.5,0),(2.5,1)
$$

and

$$
(2.5,0),(3.5,1),(0.5,1) \text {. }
$$

The number of connected components of this graph (two for Figure 13) equals $v(\widetilde{\sigma})$. This can be seen as follows: The interior of the polygons $P, P^{\prime}$ correspond to the half-planes $y<0$ and $y>1$. Vertices of $P, P^{\prime}$ correspond to the points

$$
(0.5,0),(1.5,0), \ldots,(n+0.5,0),(0.5,1),(1.5,1), \ldots,(n+0.5,1)
$$

where the pairs of points $(0.5,0),(n+0.5,0)$ and $(0.5,1),(n+0.5,1)$ have to be identified (achieved by additional arcs joining these points). The segments $(i, 0),(|\widetilde{\sigma}(i)|, 1)$ represent glued edges with dotted arcs joining vertices identified under gluing.

Let us analyze the local situation around a block $B$ of $\sigma$. The (internal) dotted arcs associated to lines of $B$ connect the four boundary points adjacent to $B$ in one of the three ways depicted in Figure 14. The proof is now obvious since each of these three situations is invariant under vertical and horizontal reflections.
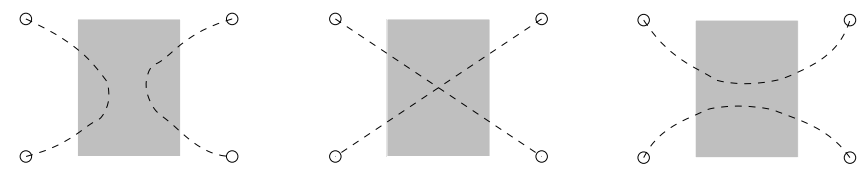

Figure 14: Three local situations around a block

We leave the easy proofs of Corollaries 8.2 and 8.3 to the reader.

Remark 8.4 Many similar invariants of spindle-permutations, up to spindle-equivalence, can be defined similarly by considering a set $\mathcal{S}$ of subsets of lines in $\sigma$ which is defined in a topological way (for example triplets of lines of a given isotopy class, 
subsets of $k$ lines defining a subspindle in a set of prescribed spindle-equivalence classes, etc) and by considering all sign sequences $\left(\epsilon_{1}, \ldots, \epsilon_{n}\right) \in\{ \pm 1\}^{n}$ such that $\left\{i \mid \epsilon_{i}=-1\right\} \in \mathcal{S}$. Then, the corresponding sum $\sum z^{v\left(\sigma_{\epsilon}\right)}$ is of course well-defined for spindles and thus for spindle-permutations, up to equivalence.

The following two questions are natural:

(1) Corollary 1.3 shows that the (complete) spindlegenus factorizes through switching classes associated to spindles. Can the spindlegenus (or some related invariant) be extended to all switching classes?

(2) Is there a natural extension of the invariant defined by the (complete) spindlegenus to skew configurations which are not spindles?

A positive answer to Question (1) above yields such an extension but a solution to Question (2) does perhaps not factorize through switching classes.

\section{Acknowledgments}

We express our thanks to several anonymous referees. Two referees commented on a previous version (consisting mainly of a flawed proof of Theorem 1.1) helpfully. Among other improvements, a referee of the present version simplified the proof of Proposition 6.7 substantially. Another referee provided accurate historical information.

The first author thanks also D Matei for his interest and valuable comments and acknowledges support from the Swiss National Science Foundation gratefully.

The second author wishes to thank the Institut Fourier where most of this work was done and Mikhail Zaidenberg for hosting his stay and acknowledges a partial support by the Chateaubriand postdoctoral fellowship funded by the French government.

\section{References}

[1] A Borobia, V F Mazurovskiĭ, On diagrams of configurations of 7 skew lines of $\mathbb{R}^{3}$, from: "Topology of real algebraic varieties and related topics", Amer. Math. Soc. Transl. Ser. 2 173, Amer. Math. Soc., Providence, RI (1996) 33-40 MR1384306

[2] A Borobia, V F Mazurovskiü, Nonsingular configurations of 7 lines of $\mathbb{R P}^{3}$, J. Knot Theory Ramifications 6 (1997) 751-783 MR1483785

[3] H Crapo, R Penne, Chirality and the isotopy classification of skew lines in projective 3-space, Adv. Math. 103 (1994) 1-106 MR1257213 
[4] Y V Drobotukhina, An analogue of the Jones polynomial for links in $\mathbb{R P}^{3}$ and a generalization of the Kauffman-Murasugi theorem, Algebra i Analiz 2 (1990) 171-191 MR1073213

[5] B Grünbaum, Arrangements and spreads, American Mathematical Society Providence, R.I. (1972) MR0307027

[6] S I Khashin, Projective theory of graphs, and configurations of lines, Zap. Nauchn. Sem. S.-Peterburg. Otdel. Mat. Inst. Steklov. (POMI) 231 (1995) 309-322, 328 (1996) MR1434302

[7] S I Khashin, V F Mazurovskiĭ, Stable equivalence of real projective configurations, from: "Topology of real algebraic varieties and related topics", Amer. Math. Soc. Transl. Ser. 2 173, Amer. Math. Soc., Providence, RI (1996) 119-140 MR1384313

[8] C L Mallows, N A Sloane, Two-graphs, switching classes and Euler graphs are equal in number, SIAM J. Appl. Math. 28 (1975) 876-880 MR0427128

[9] W S Massey, Algebraic topology: an introduction, Springer, New York (1977) MR0448331

[10] D Matei, A I Suciu, Homotopy types of complements of 2-arrangements in $\mathbb{R}^{4}$, Topology 39 (2000) 61-88 MR1710992

[11] V F Mazurovskiı̌, Configurations of six skew lines, Zap. Nauchn. Sem. Leningrad. Otdel. Mat. Inst. Steklov. (LOMI) 167 (1988) 121-134, 191 MR964261 Translation in J. Soviet Math. 52 (1990) 2825-2832

[12] V F Mazurovskiü, Configurations of at most 6 lines of $\mathbb{R} \mathbb{P}^{3}$, from: "Real algebraic geometry (Rennes, 1991)", Lecture Notes in Math. 1524, Springer, Berlin (1992) 354-371 MR1226266

[13] J Pach, R Pollack, E Welzl, Weaving patterns of lines and line segments in space, Algorithmica 9 (1993) 561-571 MR1221818

[14] J J Seidel, Graphs and two-graphs, from: "Proceedings of the Fifth Southeastern Conference on Combinatorics, Graph Theory and Computing (Florida Atlantic Univ., Boca Raton, Fla., 1974)", Congressus Numerantium X, Utilitas Math., Winnipeg, Man. (1974) 125-143 MR0364028

[15] N J A Sloane, The On-Line Encyclopedia of Integer Sequences Available at http:// oeis.org/

[16] O Y Viro, Topological problems on lines and points of three-dimensional space, Dokl. Akad. Nauk SSSR 284 (1985) 1049-1052 MR810058

[17] O Y Viro, Y V Drobotukhina, Configurations of skew-lines, Algebra i Analiz 1 (1989) 222-246 MR1027468 arXiv:math.GT/0611374

[18] T Zaslavsky, Glossary of signed and gain graphs and allied areas, Electron. J. Combin. 5 (1998) Dynamic Surveys 9, 41 pp. MR1744870 
Institut Fourier, BP 7438402 Saint-Martin D'Heres Cedex, France

Department of Applied Mathematics, School of Sciences, Holon Institute of Technology 52 Golomb Street, PO Box 305, 58102 Holon, Israel

roland.bacher@ujf-grenoble.fr, garber@hit.ac.il

Proposed: Dave Gabai

Received: 29 September 2005

Seconded: Joan Birman and Jean-Pierre Otal

Revised: 14 November 2006 